\title{
Combined Microsurgical, Endoscopic and Neuronavigation Assisted Transseptal- Transsphenoidal Resection of Pituitary Tumors
}

\author{
Mirza Pojskić ${ }^{1}$, Alisa Arnautovic ${ }^{2}$, Marko Kovacevic $^{3}$, Neal S. Beckford ${ }^{4}$, Mohammad N. Qureshi ${ }^{5}$, James Linder ${ }^{6}$, \\ Kenan I. Arnautovićc ${ }^{7,8}$ \\ ${ }^{1}$ Department of Neurosurgery, University of Marburg, Marburg, Germany, ${ }^{2}$ George Washington University School of \\ Medicine, Washington, DC, USA, ${ }^{3}$ Department of Neurosurgery, Osijek University, Osijek, Croatia, ${ }^{4}$ ENT Associates, \\ Memphis, TN, USA, ${ }^{5}$ Endocrine and Diabetes Clinic, Memphis, TN, USA, ${ }^{6}$ Ophthalmology Group of Midsouth, Memphis, \\ TN, ${ }^{7}$ Semmes Murphey Neurologic \& Spine Institute, Memphis, TN, USA, ${ }^{8}$ Department of Neurosurgery, University of \\ Tennessee Health Science Center, Memphis, TN, USA
}

Correspondence: kenanarnaut@yahoo.com; Tel.: + 1901522 2671; Fax.: + 19012592011

Received: 13 June 2020; Accepted: 30 August 2020

\begin{abstract}
Objective. To describe the technical nuances of multimodal transseptal-transsphenoid surgery for pituitary tumors using a combination of microneurosurgery, neuroendoscopy, and electromagnetic neuronavigation. Materials and Methods. A transnasal approach to the sella is performed endoscopically and widely exposed by an otolaryngologic surgeon. Surgery is next performed by the neurosurgeon with microscope and neuronavigation for microsurgical resection of pituitary tumors. Neuroendoscope is also used at the end of surgery to confirm tumor resection and inspect operative site. During surgery, the patient's head, angle and height of the microscope, and position of the table are repositionable to allow for multiple angle views. Abdominal fat harvested prior to the procedure is used to ensure cerebrospinal fluid seal. Results. The senior author (KIA) has used the combined approach with 84 consecutive patients. Radical resection was achieved in 66 patients, subtotal in 11, and partial in 7. There were no perioperative complications. Six patients experienced postoperative transient diabetes insipidus. The pituitary gland and stalk were preserved in all cases. Visual symptoms were improved in $78 \%$ and endocrinological symptoms in $56 \%$ of cases. Conclusion. This combined approach is safe and effective. It increases the efficacy and radicality of surgical resection, helps to preserve the pituitary gland, and improves and resolves preoperatively altered patient hormonal function and impaired vision. It also reduces complications, provides less postoperative pain and discomfort, reduces the surgery time, and enables a shorter hospital-stay.
\end{abstract}

Key Words: Pituitary Tumor - Combined Microsurgical and Endoscopic • Transseptal - Transsphenoid Approach - Electromagnetic Neuronavigation.

\section{Introduction}

Transsphenoidal microsurgical resection has been long established as the gold standard treatment for pituitary tumors. Use of the endoscope has gained increasing popularity in the past 20 years and has been described by its advocates as being less invasive and providing better visualization of the surgical field than the microscope (especially "around the corner"), except for sacrifice of the middle turbinate $(1,2)$.

Although endoscopic and microscopic techniques for pituitary lesion resection are often portrayed as competitive approaches, we believe they are complementary and that both should be used to optimize the minimally invasive surgical technique. The addition of electromagnetic neuronavigation, which allows free head movement, enhances the benefit of these techniques. Furthermore, a combined interdisciplinary transseptaltranssphenoid surgery (TTA) to the sella, which involves a neurosurgeon and an otolaryngologic (ENT) surgeon, minimizes the complication rate.

All techniques described above have been used previously and are well described in the literature 
with good results. Any 2 of the 3 techniques used in combination have also been described previously in the literature. However, the use of all 3 techniques combined have shown good results in our experience.

Herein, we describe our experiences and original technical steps of combining these techniques in the surgical workflow.

\section{Materials and Methods}

Appropriate Institutional Review Board (IRB) Approval was obtained prior to study. Preoperative evaluation included detailed neurological, neuroradiological, ophthalmological, and endocrine assessment. We included all patients presenting with sellar or supra/parasellar tumors who were operated on using this technique. Magnetic resonance imaging (MRI) of the head was obtained with $2 \mathrm{~mm}$ thin cuts, using navigation system protocol to accurately delineate the sellar region and surrounding structures (pituitary imaging protocol [PIP]). The MRI-Pituitary protocol +/- contrast was done prior to surgery, 6 weeks after the surgery, and every year subsequently. In the event that the patient cannot have an MRI, a computerized tomography (CT) scan with contrast (PIP) can be used. A neuro-radiologist evaluated MRI scans independently and their reports were used for assessment. Full endocrinological pituitary hormonal workup was done by a neuro-endocrinologist (MNQ) prior to surgery, immediately postoperatively in the hospital, and then every 6 and 12 months, respectively, or more frequently as needed. Full ophthalmologic workup was done by a neuro-ophthalmologist (JL) preoperatively, and then every 6-12 months postoperatively, or as needed.

\section{Operative Technique}

The patient was placed supine on the operating table with the head elevated approximately $15^{\circ}$ on a donut sponge headrest. The head was unattached and free to move in all directions to improve visualization as rigid fixation limits intraoperative manipulation of the patient's head.

An AxiEM neuronavigation system (Medtronic, Minneapolis, MN, USA) was used for electromagnetic navigation. A patient tracker (cranial dynamic reference frame) was put on the ipsilateral forehead. Once the patient was registered according to the MRI or CT imaging loaded into the system, a tracker was mounted on the endoscope rod using the tip of the rod as a navigation tool. Likewise, surgical instruments can be registered and calibrated.

Neuronavigation enabled precise localization, especially in complicated cases that transgress compartments, for recurrent cases, or in patients who underwent previous nasal surgeries in which
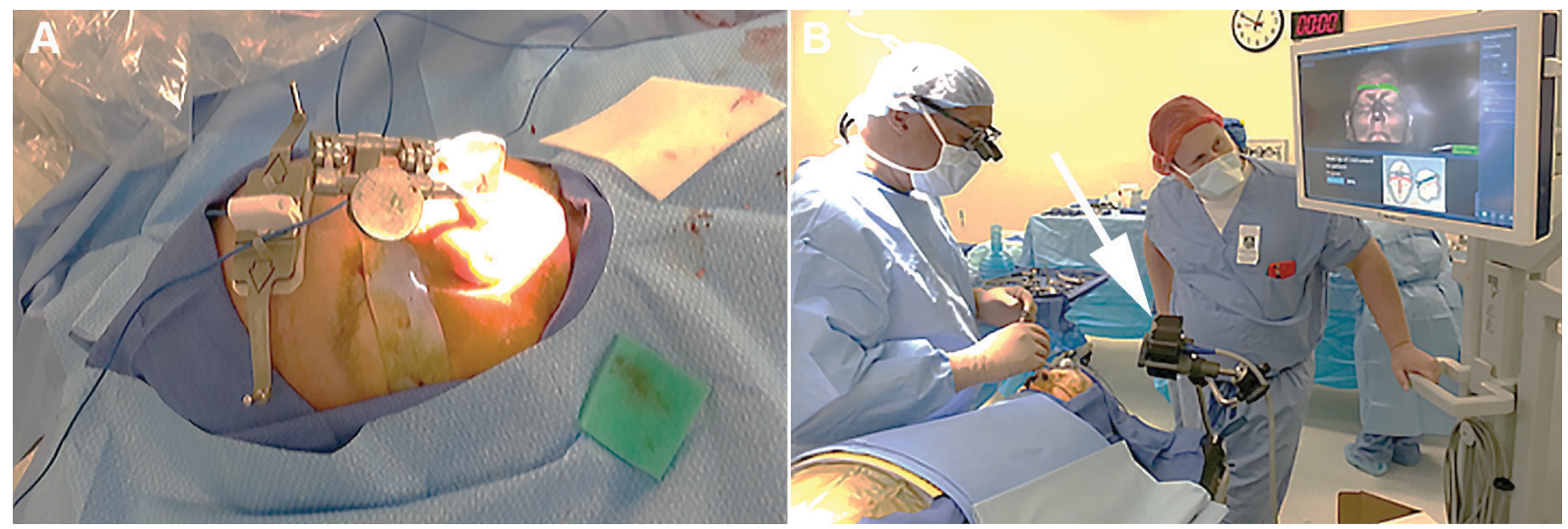

Figure 1. Operative setting for transsphenoidal combined microsurgical and endoscopic approach. (A) The patient tracker is placed on the forehead. (B) The AxiEM magnetic field emitter (white arrow) was fixed on the side of the surgical table directly and adjacent to the head of patient to obtain a stable magnetic field covering the head. The patient is registered according to MRI images, which are loaded into the system. 
the anatomy was distorted due to scar tissue, there was a narrow distance between the carotid arteries, and the sphenoid sinus was poorly pneumatized (Figure 1).

A transnasal approach to the sella was performed by the ENT surgeon (NSB). Before the surgery, the nasal mucosa of the cartilaginous septum was infiltrated unilaterally with a $1 \%$ lidocaine solution containing $0.05 \%$ of adrenaline to reduce bleeding and facilitate dissection of mucosa from the septum. The patient's lower face was prepped and draped in sterile fashion. At the same time, the right abdominal region was prepped and draped for an abdominal fat graft harvest used to prevent cerebrospinal fluid (CSF) leak when closing the surgery site.

A hemitransfixion incision was made just posterior to the columella (usually on the left), and the septal mucosa was dissected from the adjacent cartilage and bone. The contiguous floor of nose mucosa can be elevated as well for better exposure. The bony/cartilaginous junction was divided and the mucoperiosteum was elevated on the contralateral side. The perpendicular plate of the ethmoid and the vomer bone were removed, exposing the sphenoid rostrum. Visualization for this portion of the procedure was facilitated with the use of a nasal endoscope (Karl Storz, Tuttlingen, Germany or Zomed/Medtronic, Minneapolis, MN, USA). A pituitary speculum was then inserted in the nose, separating the septal mucosa, facilitating optimal exposure of the rostrum, and facilitating bimanual instrumentation. The sphenoid sinus ostia were identified and used as a point of entry into the sphenoid sinus. The entire rostrum was then removed using Kerrison rongeurs and endoscopic sinus instruments, thus maximizing exposure of the operative field. Although the neuronavigation system helps identify the trajectory to the sellar floor, neuroanatomical knowledge of the region such as the carotid prominences - is essential for establishment of safe working zone (Figure 2).

When the sellar floor was reached and bone widely opened, surgery was next performed by a neurosurgeon with microsurgical technique. At this point, the microscope was brought in (Kinevo

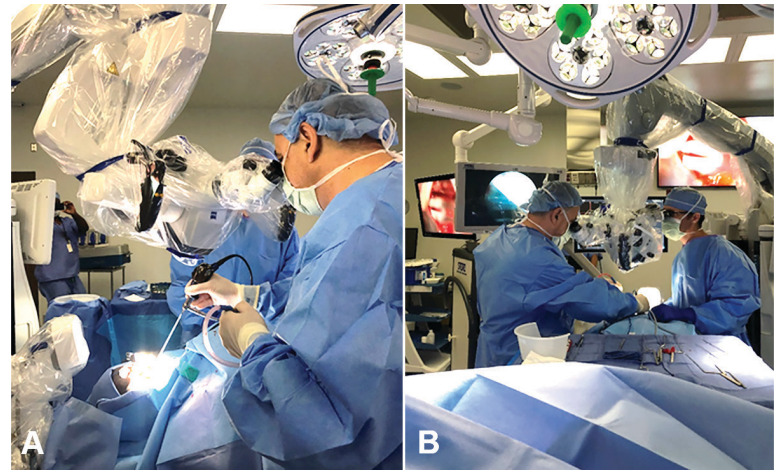

Figure 2. Operative setting with simultaneous use of $(A)$ endoscope and (B) microscope with electromagnetic-navigation guided resection of pituitary tumor.

900 or Pentero 900, Zeiss, Oberkochen, Germany). The surface of the cavernous sinus was exposed bilaterally as needed. The dura was opened in an "X" shape. Microsurgical resection of the pituitary tumor was usually performed using curettes of various angles and sizes, bipolar electrocautery, and micro-forceps.

Resection begins with a ringed curette that loosened the tissue. We prefer usage of $30^{\circ}$ angled curettes. It is advised to remove the laterally situated regions of the tumor first as well as lower ones, followed by the more central segments in order to avoid entrapment of the lateral portions by prematurely descending the diaphragma sellae. Superior dissection should be performed only when the tumor is freed inferiorly. Controlled suction along with bipolar forceps, micro pituitary rongeurs, micro-dissectors, and curettes were used interchangeably using 2 hands technique. If the suprasellar part of the tumor does not prolapse into view following resection of the intrasellar part, Valsalva maneuver can be used to facilitate descent of the residual tumor. The endoscope with a multiple angle lens can be used to locate any lateral fragments.

Throughout the surgery, repositioning the patient's head, angle and height of the microscope, the position of the table with microscope magnification, as well as the use of the endoscope and electromagnetic navigation with a registered pointer and instrument, allowed for a full and optimal view of the surgical field and exact localiza- 
tion. This range of motion and visualization outreached the view provided by endoscope alone. If and when residual tumor was identified by endoscope, microsurgical or endoscopic technique was used to remove it as deemed appropriate intraoperatively.

If CSF leak was noted, the abdominal fat graft harvested at the beginning of surgery was placed on the sellar floor intradurally. The reconstruction can be reinforced with fibrin glue. The speculum was removed and the septum pushed into the midline from the other nostril and mucosal flap reflected over the septum and secured with absorbable suture. Endonasal trumpets, that allow breathing through the nostrils but remain unseen when looking at patient, were placed bilaterally and secured with permanent sutures. They were removed on the fourth day during an outpatient visit following surgery. The patient usually leaves the hospital on the first day following surgery after overnight monitoring for diabetes insipidus. Operative Procedure YouTube Video Clip Link: https://youtu.be/AvL03Jn1DS4

\section{Results}

In this personal series of the senior author (KIA), the combined approach was used on 84 consecutive patients (January 2011-December 2017) in cooperation with an ENT surgeon and a multidisciplinary team (endocrinology, ophthalmology). The series was continued up to the present. The mean age of the patients was 55 years (range, 16-70) with 51 male and 33 female patients. Mean follow-up was 37 months. There were 76 pituitary adenomas, 4 Rathke's cleft cyst, 1 clival chordoma, 1 renal cell carcinoma metastasis, and 2 cases of lymphocytic hypophysitis. Radical resection was achieved in 66 patients, subtotal resection in 11 patients, and partial resection/biopsy in 7 patients (based on neuroradiology reports). The mean length-of-stay was
3 days. There were no perioperative complications. Six patients experienced postoperative transient diabetes insipidus. CSF leak possibility was eliminated with the placement of fat graft. The pituitary gland and stalk were preserved in all cases. Visual symptoms were improved in $78 \%$ and endocrinological symptoms in $56 \%$ of the cases based on neuro-ophthalmology and neuro-endocrinology assessment. Six cases that had substantial supra/ parasellar tumor extension were subsequently operated on via craniotomy as a second stage. We did not have any postoperative CSF leaks. There were no postoperative transient or permanent olfactory deficits (Figures 3, 4, 5, and 6).

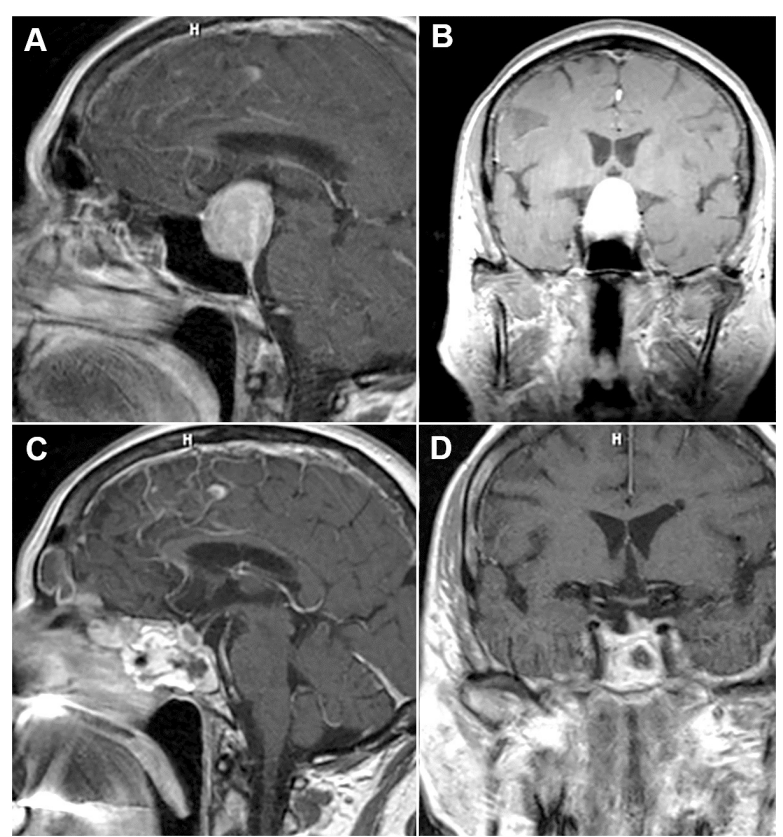

Figure 3. A woman in her late 70s presented with severe headache, VI nerve palsy, and visual decline. Combined transsphenoidal resection for hormone-inactive macroadenoma was performed. Preoperative (A) T1 sagittal and (B) T1 coronal post-contrast MRI of the head shows large intra, para, and suprasellar tumor with compression of optic chiasm and infiltration of cavernous sinus. Postoperative (C) sagittal and (D) coronal post-contrast T1 MRI of the head shows complete resection of the tumor with intact pituitary gland/stalk (note the fat graft in the sphenoid sinus-SS). The patient recovered fully her vision and double vision. 


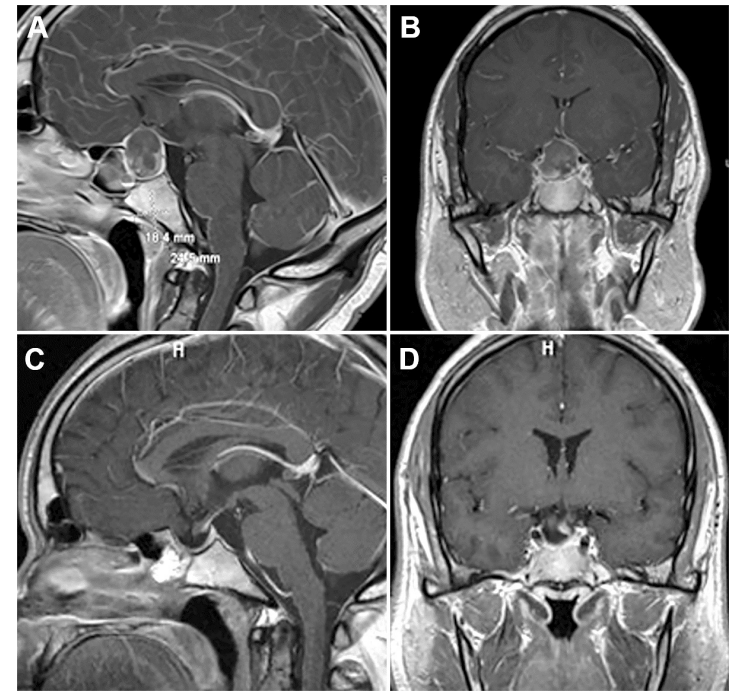

Figure 4. A man in the fifth decade of life experienced a sudden onset of severe headache and acute blindness. Combined trans-sphenoidal approach for pituitary apoplexy was performed. Preoperative (A) T1 sagittal and (B) coronal post-contrast MRI of the head shows large intra, para, and suprasellar hematoma with compression of optic chiasm and infiltration of cavernous sinus. Postoperative T1 (C) sagittal and (D) coronal post-contrast MRI of the head shows complete resection of the lesion with intact pituitary gland/ stalk (note the small fat graft in the SS. Visual symptoms recovered following surgery (see also YouTube link).

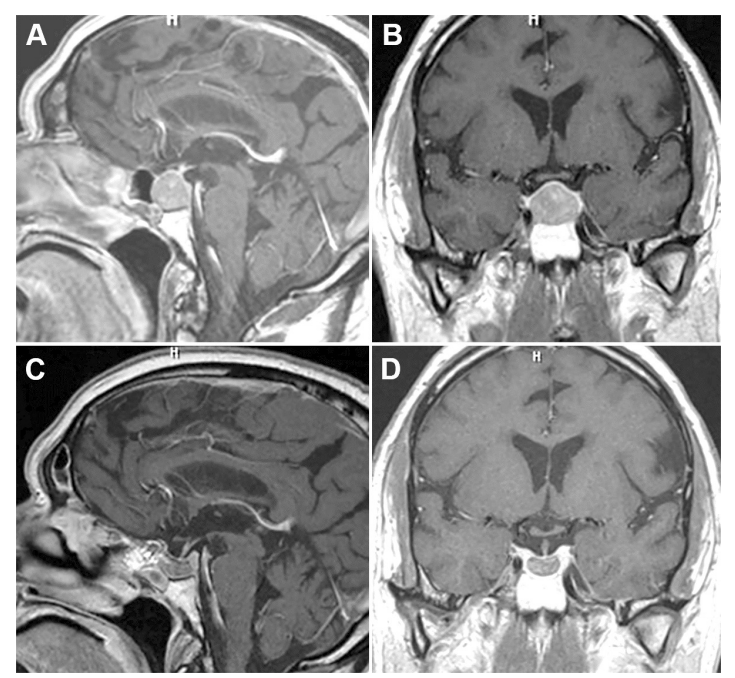

Figure 6. A man in his early 60s presented with acromegaly. Combined transsphenoidal approach was performed. Preoperative (A) sagittal (B) coronal post-contrast T1 MRI of the head shows intra, and supra-sellar tumor with intratumoral hemorrhage and compression of optic chiasm. Postoperative (C) sagittal and (D) coronal post-contrast MRI of the head shows complete resection of the tumor with intact pituitary gland/stalk. Visual symptoms recovered with remission of acromegaly.

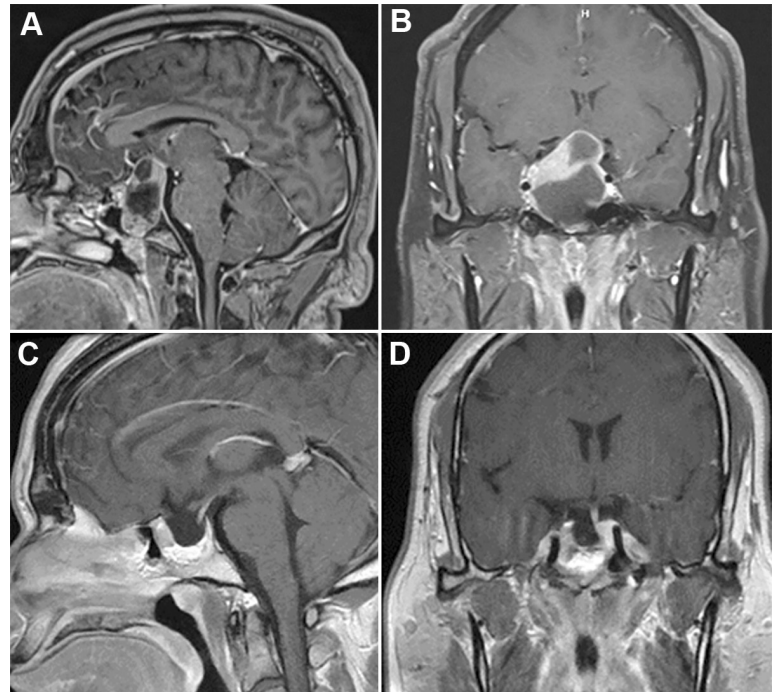

Figure 5. A man in his late 20s experienced a sudden onset of severe headache and blurred vision. Combined transsphenoidal approach for giant prolactinoma with intratumoral hemorrhage was performed. Preoperative (A) T1 sagittal and (B) T1 coronal post-contrast MRI of the head shows large intra, para, and suprasellar tumor with intratumoral hemorrhage with compression of optic chiasm and infiltration of cavernous sinus. Postoperative (C) sagittal and (D) coronal post-contrast T1 MRI of the head shows complete resection of the tumor and intact pituitary gland/ stalk. Visual symptoms recovered and prolactin serum level improved significantly (preoperative, $1000 \mathrm{nmol} / \mathrm{L}$ vs. postoperative, $200 \mathrm{nmol} / \mathrm{L}$ ) following surgery.

\section{Discussion}

\section{Microsurgery vs endoscopic surgery and our experiences with combination}

We utilized an original combination of a microsurgical and endoscopic approach combined with Axiem neuro-navigation to remove pituitary tumors in order to best employ the surgical and technical advantages of all 3 techniques.

The transsphenoidal microsurgical approach has been considered to be the gold standard for operative treatment of pituitary tumors (3). The technique introduced by Schoffler was modified by Hardy and Guiot in the 1960s with the introduction of microscopy and fluoroscopy and was used for many years. Guiot was the first surgeon to describe using endoscopes in pituitary surgery (4). Over time, the endoscopic approach gained popu- 
larity and may have been more frequently used. It was believed to be less invasive and allowed for seeing around different angles or "around the corner." It has better visualization of the close-up surgical field, an enlarged working angle, and an increased panoramic view. Four disadvantages of the endoscopic technique are noted: the possible need to remove the normal middle turbinate, relatively high frequency of temporary or permanent loss of smell, 2D visual experience, and the definite need of additional simultaneous participating surgeons. Although there are newly developed 3-dimensional (3D) models of the endoscope, they clearly lack the 3D advantage of microsurgical technique. Gross total resection of macroadenomas with parasellar extension was reported to be significantly enhanced by the endoscopic approach (5$7)$. The endoscopic approach has also been reported to show better results in invasive adenomas (8), lowers the incidence of new hypopituitarism after surgery $(2,6,9)$, has better intraoperative identification and preservation of pituitary gland (2), and results in shorter hospital stay and less post-op pain (10).

A significant difference in the complication rates between endoscopic and microscopic technique, however, was not found in recent studies (10-12). Also, these studies on patients who underwent transsphenoidal resection for Cushing's disease (13) and acromegaly (14) found that remission and recurrence rates were the same among patients who underwent endoscopic surgery versus microscopic surgery. Both techniques have been found to be comparable for infradiaphragmatic craniopharyngiomas, while the endoscopic approach yields better results for supradiaphragmatic lesions (15). However, a large retrospective review of 1054 patients with microsurgically resected craniopharyngioma revealed good patient outcomes without more limitations on each individual tumor with distinct features, despite the impact of recent endoscopic techniques (16). On the contrary, a recent literature review has shown that vascular complications and CSF fistulas were reduced with microsurgery compared with endoscopy (17). As a matter of fact, these 2 approaches may have driven neurosurgeons into the 2 competing camps that exclusively utilizes one procedure or the other.

Although the combined use of a microscope and endoscope for pituitary surgery was described by Bush and Halves in 1978 (18), we have hypothesized that using a combination of microscope and endoscope along with neuronavigation can capture the best advantages of all 3 techniques. An inspiring report by Al-Mefty et al. described the use of combined approach to yield benefits of both techniques (4) as the 2 approaches became more competitive among neurosurgeons over the years.

Combined approach to pituitary lesions has been previously reported. The term "combined approach" described either a combined endoscopic and microscopic transsphenoidal approach to pituitary lesions, or a combined transcranial and transsphenoidal approach. The additional support by neuronavigation for microsurgical or endoscopic approach has also been published (3, 4, 19). Combined endoscopic and microscopic management of pituitary region tumors has also been described in a technical note for pediatric surgery $(20)$, in case reports $(21,22)$ and in few retrospective studies $(4,23,24)$. In the pioneering work of Helal et al. in which 37 patients were treated with a combined approach, the additional use of an endoscope was highly beneficial as hidden areas could be visualized in $84 \%$ of the patients, and tumor residues were detected in $40.5 \%$ (23).

The transition from microsurgical to endoscopic approach has also been previously published (25). Laws et al. recommended that the operating microscope should always be balanced and readily available since up to $14 \%$ of endoscopic surgeries were converted from an endoscopic to microscopic approach due to tissue hypertrophy, atypical nasal airway, scar tissue, complex sphenoid sinus anatomy, mucosal bleeding, need for binocular vision in extended approach, and technical problems with the endoscope. Most of the studies, however, described the results of either microscopic or endoscopic surgery and provided a comparison of the 2 methods. According to 2 literature reviews, the endoscopic approach may be associated with a 
higher rate of gross tumor movement and a lower risk of postoperative complications for treating a nonfunctioning pituitary adenoma when compared with a microscopic approach, although the rate of permanent diabetic insipidus and meningitis remains the same $(26,27)$. An additional single institution study of 137 patients found no differences between the 2 approaches (28). A national database study of more than 30.000 patients noted a significant increase in the endoscopic surgery group over time, yet rates of gross total resection, need for adjuvant therapy, and short-term mortality were similar (29). No differences in complication rates between endoscopic and microscopic surgery were observed in a large single institution retrospective study of 1153 patients (30).

As noted above, both endoscopic and microscopic surgeries have yielded similarly good outcomes in numerous reports. Our intention was to combine all 3 techniques simultaneously to both add and multiply the benefits of all in a single procedure. We believe that one of the clear advantages of the microscopic technique compared to the endoscopic approach is preservation of all nasal and paranasal anatomical structures. Furthermore, the surgeon can freely use both hands while applying microsurgical techniques when dissecting the tumor. Also, a single surgeon controls the situation the entire time as opposed to having 2 surgeons involved in the endoscopic approach. A microscope provides magnification, illumination, $3-\mathrm{D}$ visualization, communication with operating room personnel (via microscope monitor), and intraoperative recording, which can be used for educational purposes to teach trainees.

We utilized an endoscope at the end of surgery $\left(0^{\circ}, 30^{\circ}, 60^{\circ}, 70^{\circ}\right)$ to carefully inspect the resection cavity, find any unnoticed residual tumor, and verify anatomy and lack of complications. Furthermore, our combined approach provided preservation of all anatomic structures, no evidence of postoperative CSF leak and no evidence of postoperative transient or permanent olfactory loss thus minimizing postoperative discomfort. Overall, our experiences indicate that both microsurgical and endoscopic techniques have merits and should be used as complementary, rather than competing, techniques (32).

\section{Neuronavigation in Transsphenoidal Surgery}

Axiem neuronavigation obviates the need for rigid head fixation. We consider the ability to freely move the head to the desired position intraoperatively to be very important. This freedom of movement enables the microscope view to be adjusted in multiple directions, enhancing the visualization capabilities of microscope itself. We found that one of the benefits versus optoelectronic navigation is that there are no line-of-sight problems when tracking instruments, and accuracy is comparable to standard navigation. This has been reported by other authors, too (31). A drawback of conventional neuronavigation, however, is the necessity of focusing on 2-dimensional images in 3 planes at the same time to determine one's position in the operating field. Several reports described the use of neuronavigation in combination with microsurgical or endoscopic approach alone, but only a few reports have described the use of a combined microscopic and endoscopic approach with use of neuronavigation $(3,4,19)$. Only Al-Mefty et al. (4) described the use of a free-head navigation technique in combination with microscope and endoscope. Our additional observation is that the use of neuro-navigation obviates the use of bulky $\mathrm{C}$-arm fluoroscopy and the subsequent health hazards of intraoperative radiation to operative room personnel and surgeons.

\section{Conclusion}

Our experiences utilizing the original combination of microsurgical and endoscopic endonasal transsphenoidal approach and using neuronavigation indicate that it is a safe and effective practice for the resection of pituitary tumors. Our results indicate that it may increase the efficacy of resection in all relevant outcome parameters, such as increasing the radicality of surgical resection, helping to preserve the pituitary gland, and helping to improve and resolve preoperatively altered 
patient hormonal function and impaired vision. Furthermore, the combined approach reduces complications (CSF leak and postoperative olfactory loss), provides less postoperative pain and discomfort, reduces the surgery time, and enables a shorter hospital-stay.

\section{What Is Already Known on this Topic:}

The utilization of microsurgical or endoscopic technique for the resection of pituitary tumors have both been proven to be effective. However, these 2 techniques are presently used competitively and not simultaneously. This antagonism deprives surgeons to use the benefits of both techniques simultaneously.

\section{What this Study Adds:}

Utilizing the original combination of microsurgical and endoscopic endonasal transsphenoidal approach and using neuronavigation proves to be a safe and effective practice for the resection of pituitary tumors. Our results on a relatively large series of patients indicate that it may increase the efficacy of resection in all relevant outcome parameters: increasing the radicality of surgical resection, helping to preserve the pituitary gland, and helping to improve and resolve preoperatively altered patient hormonal function and impaired vision. Furthermore, the combined approach reduces complications, provides less postoperative pain and discomfort, reduces the surgery time, and enables a shorter hospital-stay. Finally, combination of these 3 techniques into one accumulates and enhance their singular benefits.

Acknowledgement: The authors wish to thank Andrew J. Gienapp (Neuroscience Institute, Le Bonheur Children's Hospital and Department of Neurosurgery, University of Tennessee Health Science Center, Memphis, TN, USA) for copy and technical editing, and preparation of the manuscript and figures for publishing.

Authors' Contributions: Conception and design: MP, AA, $\mathrm{MK}, \mathrm{NB}, \mathrm{MQ}$, JL and KA; Acquisition, analysis and interpretation of data: MP, MK and KA; Drafting the article: MP, AA, MK, NB, MQ, JL and KA; Revising it critically for important intellectual content: MP, MK, NB, MQ, JL and KA; Approved final version of the manuscript: KA.

Conflict of Interest: The authors declare that they have no conflict of interest.

\section{References}

1. Kutlay M, Gönül E, Düz B, Izci Y, Tehli O, Temiz C, et al. The use of a simple self-retaining retractor in the endoscopic endonasal transsphenoidal approach to the pituitary macroadenomas: technical note. Neurosurgery. 2013;73(2 Suppl Operative):ons206-9; discussion ons209-10.

2. Linsler S, Senger S, Hero-Gross R, Steudel WI, Oertel J. The endoscopic surgical resection of intrasellar lesions conserves the hormonal function: a negative correlation to the microsurgical technique. J Neurosurg Sci. 2018 Mar 28. Epub ahead of print.

3. Buchfelder M, Schlaffer SM, Zhao Y. The optimal surgical techniques for pituitary tumors. Best Pract Res Clin Endocrinol Metab. 2019;33(2):101299.

4. Al-Mefty O, Pravdenkova S, Gragnaniello C. A technical note on endonasal combined microscopic endoscopic with free head navigation technique of removal of pituitary adenomas. Neurosurg Rev. 2010;33(2):243-8; discussion 8-9.

5. Trevisi G, Vigo V, Morena MG, Grieco DL, Rigante M, Anile C, et al. Comparison of Endoscopic Versus Microsurgical Resection of Pituitary Adenomas with Parasellar Extension and Evaluation of the Predictive Value of a Simple 4-Quadrant Radiologic Classification. World Neurosurg. 2019;121:e769-74.

6. Pal'a A, Knoll A, Brand C, Etzrodt-Walter G, Coburger J, Wirtz CR, et al. The Value of Intraoperative Magnetic Resonance Imaging in Endoscopic and Microsurgical Transsphenoidal Pituitary Adenoma Resection. World Neurosurg. 2017;102:144-50.

7. Lenzi J, Lapadula G, D’amico T, Delfinis CP, Iuorio R, Caporlingua F, et al. Evaluation of trans-sphenoidal surgery in pituitary GH-secreting micro- and macroadenomas: a comparison between microsurgical and endoscopic approach. J Neurosurg Sci. 2015;59(1):11-8.

8. Pablo A, Sofia B, Maximiliano T, Patricia FD, Alvaro C, Claudio Y, et al. Endoscopic versus Microscopic Pituitary Adenoma Surgery: A Single-center Study. Neurol India. 2019;67(4):1015-21.

9. Hlaváč M, Knoll A, Etzrodt-Walter G, Sommer F, Scheithauer M, Coburger J, et al. Intraoperative MRI in transsphenoidal resection of invasive pituitary macroadenomas. Neurosurg Rev. 2019;42(3):737-43.

10. Cappabianca P, Cavallo LM, de Divitiis O, Solari D, Esposito F, Colao A. Endoscopic pituitary surgery. Pituitary. 2008;11(4):385-90.

11. Halvorsen H, Ramm-Pettersen J, Josefsen R, Rønning P, Reinlie S, Meling T, et al. Surgical complications after transsphenoidal microscopic and endoscopic surgery for pituitary adenoma: a consecutive series of 506 procedures. Acta Neurochir (Wien). 2014;156(3):441-9.

12. Frank G, Pasquini E, Farneti G, Mazzatenta D, Sciarretta V, Grasso V, et al. The endoscopic versus the traditional approach in pituitary surgery. Neuroendocrinology. 2006;83(3-4):240-8.

13. Qiao N. Outcome of endoscopic vs microsurgical transsphenoidal resection for Cushing's disease. Endocr Connect. 2018;7(1):R26-37.

14. Chen CJ, Ironside N, Pomeraniec IJ, Chivukula S, Buell TJ, Ding D, et al. Microsurgical versus endoscopic transsphenoidal resection for acromegaly: a systematic review of outcomes and complications. Acta Neurochir (Wien). 2017;159(11):2193-207. 
15. Cagnazzo F, Zoli M, Mazzatenta D, Gompel JJV. Endoscopic and Microscopic Transsphenoidal Surgery of Craniopharyngiomas: A Systematic Review of Surgical Outcomes Over Two Decades. J Neurol Surg A Cent Eur Neurosurg. 2018;79(3):247-56.

16. Shi X, Zhou Z, Wu B, Zhang Y, Qian H, Sun Y, et al. Outcome of Radical Surgical Resection for Craniopharyngioma with Hypothalamic Preservation: A Single-Center Retrospective Study of 1054 Patients. World Neurosurg. 2017;102:167-80.

17. Simal-Julián JA, Miranda-Lloret P, Pancucci G, Evangelista-Zamora R, Pérez-Borreda P, Sanromán-Álvarez P, et al. Microscopic versus endoscopic pituitary surgery. Neurocirugia (Astur). 2014;25(4):170-8.

18. Bushe KA, Halves E. Modified technique in transsphenoidal operations of pituitary adenomas. Technical note (author's transl) [in German]. Acta Neurochir (Wien). 1978;41(1-3):163-75.

19. Carl B, Bopp M, Voellger B, Saß B, Nimsky C. Augmented Reality in Transsphenoidal Surgery. World Neurosurg. 2019;125:e873-83.

20. Frazier JL, Chaichana K, Jallo GI, Quiñones-Hinojosa A. Combined endoscopic and microscopic management of pediatric pituitary region tumors through one nostril: technical note with case illustrations. Childs Nerv Syst. 2008;24(12):1469-78.

21. Faggin R, Pentimalli L, Grazzini M, Saetti R, Drigo P, d'Avella D. Combined endoscopic-microsurgical approach for transsphenoidal (sphenopalatine) encephalocele with an intralesional pituitary gland. Case report. J Neurosurg Pediatr. 2009;4(3):262-5.

22. Yoshida K, Toda M, Akiyama T, Takahashi S, Nishimoto M, Ozawa H, et al. Combined Endoscopic Endonasal and Video-microscopic Transcranial Approach with Preoperative Embolization for a Posterior Pituitary Tumor. World Neurosurg. 2018;119:201-8.

23. Helal MZ. Combined micro-endoscopic trans-sphenoid excisions of pituitary macroadenomas. Eur Arch Otorhinolaryngol. 1995;252(3):186-9.
24. Adeolu AA, Osazuwa UA, Oremakinde AA, Oyemolade TA, Shokunbi MT. Combined microsurgical extra-axial and transcortical transventricular endoscopic excision of parasellar tumors with ventricular extension. Ann Afr Med. 2015;14(3):155-8.

25. Laws ER, Barkhoudarian G. The transition from microscopic to endoscopic transsphenoidal surgery: the experience at Brigham and Women's Hospital. World Neurosurg. 2014;82(6 Suppl):S152-4.

26. Yu SY, Du Q, Yao SY, Zhang KN, Wang J, Zhu Z, et al. Outcomes of endoscopic and microscopic transsphenoidal surgery on non-functioning pituitary adenomas: a systematic review and meta-analysis. J Cell Mol Med. 2018;22(3):2023-7.

27. Gao Y, Zhong C, Wang Y, Xu S, Guo Y, Dai C, et al. Endoscopic versus microscopic transsphenoidal pituitary adenoma surgery: a meta-analysis. World J Surg Oncol. 2014;12:94.

28. Broersen LHA, van Haalen FM, Biermasz NR, Lobatto DJ, Verstegen MJT, van Furth WR, et al. Microscopic versus endoscopic transsphenoidal surgery in the Leiden cohort treated for Cushing's disease: surgical outcome, mortality, and complications. Orphanet J Rare Dis. 2019;14(1):64.

29. Goshtasbi K, Lehrich BM, Abouzari M, Abiri A, Birkenbeuel J, Lan MY, et al. Endoscopic versus nonendoscopic surgery for resection of pituitary adenomas: a national database study. J Neurosurg. 2020:1-9.

30. Agam MS, Wedemeyer MA, Wrobel B, Weiss MH, Carmichael JD, Zada G. Complications associated with microscopic and endoscopic transsphenoidal pituitary surgery: experience of 1153 consecutive cases treated at a single tertiary care pituitary center. J Neurosurg. 2018:1-8.

31. Sangra M, Clark S, Hayhurst C, Mallucci C. Electromagnetic-guided neuroendoscopy in the pediatric population. J Neurosurg Pediatr. 2009;3(4):325-30.

32. Pojskić M, Zbytek B, Beckford NS, Boop FA, Arnautović KI. First Report of Coexistence of two ectopic pituitary tumors: Rathke Cleft Cyst and silent adrenocorticotropic hormone adenoma. World Neurosurg. 2017; 104:1048.e11048.e7. 